\title{
A NOTE ON A UNICITY THEOREM OF K. TOHGE
}

\author{
XiaO-Min Li and Hong-Xun Yi
}

\begin{abstract}
In this paper, we deal with the problem of uniqueness of meromorphic functions sharing three values $\mathrm{CM}$, and get rid of the restriction on the hyper-orders in a unicity theorem of K. Tohge. An example is provided to show that the result in this paper is best possible.
\end{abstract}

\section{Introduction and main results}

Let $f$ and $g$ be two non-constant meromorphic functions in the complex plane. It is assumed that the reader is familiar with the standard notations of Nevanlinna's theory such as $T(r, f), m(r, f), N(r, f), \bar{N}(r, f)$ and so on, which can be found in [1]. We use $E$ to denote any set of positive real numbers of finite linear measure, not necessarily the same at each occurrence. The notation $S(r, f)$ denotes any quantity satisfying $S(r, f)=o(T(r, f))(r \rightarrow \infty, r \notin E)$.

Let $a$ be a complex number, we say that $f$ and $g$ share the value $a \mathrm{CM}$ provided $f-a$ and $g-a$ have the same zeros counting multiplicities (see [2]). We say that $f$ and $g$ share $\infty \mathrm{CM}$ provided that $1 / f$ and $1 / g$ share $0 \mathrm{CM}$. In this paper, we also need the following definition.

Definition. Let $f$ be a non-constant meromorphic function. The hyperorder of $f$, denoted $v(f)$, is defined by

$$
v(f)=\limsup _{r \rightarrow \infty} \frac{\log \log T(r, f)}{\log r} .
$$

In 1988, K. Tohge [3] proved the following theorems:

THEOREM A. Let $f$ and $g$ be two distinct transcendental meromorphic functions sharing 0,1 and $\infty \mathrm{CM}$. If $f^{\prime}$ and $g^{\prime}$ share $0 \mathrm{CM}$, then $f$ and $g$ satisfy one of the following relations:

2000 Mathematics Subject Classification: Primary 30D35.

Keywords: Meromorphic functions, shared values, hyper-orders, uniqueness theorem.

Project supported by NSFC and the RFDP.

Received July 6, 2001; revised July 4, 2002. 
(i) $f \cdot g \equiv 1$,

(ii) $(f-1)(g-1) \equiv 1$,

(iii) $f+g \equiv 1$,

(iv) $f \equiv c g$,

(v) $f-1 \equiv c(g-1)$

(vi) $[(c-1) f+1] \cdot[(c-1) g-c] \equiv-c$,

where $c(\neq 0,1)$ is a constant.

THEOREM B. Let $f$ and $g$ be two distinct transcendental meromorphic functions sharing 0,1 and $\infty \mathrm{CM}$, and let $a(\neq 0)$ be a finite complex number. If $f^{\prime}$ and $g^{\prime}$ share $a \mathrm{CM}$ and $\max \{v(f), v(g)\}<1$, then $f$ and $g$ satisfy one of the following relations:

(i) $f \cdot g \equiv 1$,

(ii) $(f-1)(g-1) \equiv 1$,

(iii) $[(c-1) f+1] \cdot[(c-1) g-c] \equiv-c$, where $c(\neq 0,1)$ is a constant.

Now it is natural to ask the following question:

Question 1. What can be said if we get rid of the condition "max $\{v(f), v(g)\}<1$ " in Theorem B?

In this paper, we shall answer Question 1 and obtain a new result. Indeed, we shall prove the following theorem:

THEOREM 1. Let $f$ and $g$ be two distinct transcendental meromorphic functions sharing 0,1 and $\infty \mathrm{CM}$, and let $a(\neq 0)$ be a finite complex number. If $f^{\prime}$ and $g^{\prime}$ share $a \mathrm{CM}$, then $f$ and $g$ satisfy one of the following relations:

(i) $f=A e^{a \omega z}, g=(1 / A) e^{-a \omega z}$, where $\omega$ satisfying $\omega^{2}=-1$, and $A(\neq 0)$ are constants;

(ii) $f=1+A e^{a \omega z}, g=1+(1 / A) e^{-a \omega z}$, where $\omega$ satisfying $\omega^{2}=-1$, and $A(\neq 0)$ are constants;

(iii) $f(z)=1 /(c-1)\left(A e^{a(c-1) \omega z}-1\right), \quad g(z)=c /(c-1)\left(1-(1 / A) e^{-a(c-1) \omega z}\right)$, where $A, c$ and $\omega$ are constants satisfying $A \neq 0, c \neq 0,1$ and $\omega^{2}=1 / c$.

It is obvious that if $f$ and $g$ satisfy the relations (i), (ii) and (iii) of Theorem 1 , then the order of $f$ is equal to 1 . By Theorem 1 we immediately deduce the following uniqueness theorem of meromorphic functions.

THEOREM 2. Let $f$ and $g$ be two transcendental meromorphic functions sharing 0,1 and $\infty \mathrm{CM}$, and suppose that $f^{\prime}$ and $g^{\prime}$ share a $\mathrm{CM}$, where $a(\neq 0)$ is a finite complex number. If the order of $f$ is not equal to 1 , then $f \equiv g$.

\section{Some lemmas}

The following notations are used throughout this paper.

Let $h$ be a non-constant meromorphic function, and let $k$ be a positive 
integer. We denote by $N_{k)}(r, 1 /(h-a))$ the counting function of $a$-points of $h$ with multiplicity $\leq k$, and denote by $N_{(k}(r, 1 /(h-a))$ the counting function of $a$-points of $h$ with multiplicity $\geq k$ (see [2]).

Let $f$ and $g$ share 0,1 and $\infty \mathrm{CM}$, we denote by $N_{0}(r)$ the counting function of the zeros of $f-g$ not containing the zeros of $f, 1 / f$ and $f-1$ (see [4] or [5]).

Lemma 1 (see [2, Lemma 9.1]). Let $f$ and $g$ be two non-constant meromorphic functions sharing 0,1 and $\infty \mathrm{CM}$. If

$$
\delta_{1)}(0, f)+\delta_{1)}(1, f)>\frac{3}{2}
$$

where

$$
\delta_{1)}(0, f)=1-\limsup _{r \rightarrow \infty} \frac{N_{1)}(r, 1 / f)}{T(r, f)}, \quad \delta_{1)}(1, f)=1-\limsup _{r \rightarrow \infty} \frac{N_{1)}(r, 1 /(f-1))}{T(r, f)},
$$

then $f+g \equiv 1$.

Lemma 2 (see [2, p. 369]). Let $F$ and $G$ be two non-constant meromorphic functions, and let

$$
\phi \equiv \frac{F^{\prime \prime}}{F^{\prime}}-\frac{G^{\prime \prime}}{G^{\prime}} .
$$

If $z_{\infty}$ is a common simple pole of $F$ and $G$, then $\phi\left(z_{\infty}\right)=0$.

Lemma 3 (see [4, Lemma 4]). Let $f$ and $g$ be meromorphic functions sharing $0,1, \infty$ CM. If $f \not \equiv g$, then

$$
N_{(2}\left(r, \frac{1}{f}\right)+N_{(2}\left(r, \frac{1}{f-1}\right)+N_{(2}(r, f)=S(r, f) .
$$

Lemma 4 (see [5, Lemma 7] or [6, Lemma 3]). Let $f$ and $g$ be two distinct non-constant meromorphic functions sharing 0,1 and $\infty \mathrm{CM}$. If $f$ is a Möbius transformation of $g$, then $f$ and $g$ satisfy one of the following relations:

(i) $f \cdot g \equiv 1$,

(ii) $(f-1)(g-1) \equiv 1$,

(iii) $f+g \equiv 1$,

(iv) $f \equiv c g$,

(v) $f-1 \equiv c(g-1)$,

(vi) $[(c-1) f+1] \cdot[(c-1) g-c] \equiv-c$,

where $c(\neq 0,1)$ is a constant.

Lemma 5 (see $\left[8\right.$, p. 120]). Let $f_{1}, f_{2}, \ldots, f_{n}$ be meromorphic functions linearly independent over the complex number field $C$ such that

$$
\sum_{i=1}^{n} f_{i} \equiv 1 .
$$


Then

$$
T\left(r, f_{j}\right)<\sum_{i=1}^{n} N\left(r, \frac{1}{f_{i}}\right)+(n-1) \sum_{\substack{i=1 \\ i \neq j}}^{n} \bar{N}\left(r, f_{i}\right)+S(r) \quad(1 \leq j \leq n),
$$

where $T(r)=\max _{1 \leq i \leq n}\left\{T\left(r, f_{i}\right)\right\}$ and $S(r)=o(T(r))(r \rightarrow \infty, r \notin E)$.

Lemma 6 (see [2, Theorem 1.62] or [10, Theorem 1]). Let $f_{1}, f_{2}, \ldots, f_{n}$ be non-constant meromorphic functions, and let $f_{n+1}(\not \equiv 0)$ be a meromorphic function such that

$$
\sum_{i=1}^{n+1} f_{i} \equiv 1
$$

If there exists a subset $I \subseteq R^{+}$satisfying mesI $=\infty$ such that

$$
\sum_{i=1}^{n+1} N\left(r, \frac{1}{f_{i}}\right)+n \sum_{\substack{i=1 \\ i \neq j}}^{n+1} \bar{N}\left(r, f_{i}\right)<(\lambda+o(1)) T\left(r, f_{j}\right) \quad(r \rightarrow \infty, r \in I, j=1,2, \ldots, n),
$$

where $\lambda<1$. Then $f_{n+1} \equiv 1$.

Remark. Lemma 6 plays an important role for the proof of Theorem 1. Now we give a simple proof of Lemma 6. Suppose that

$$
\sum_{j=1}^{n} f_{j} \not \equiv 0
$$

Without loss of generality, let

$$
\sum_{j=1}^{n} f_{j} \equiv \sum_{i=1}^{k} a_{i} f_{i}
$$

where $f_{1}, f_{2}, \ldots, f_{k}(1 \leq k \leq n)$ are linearly independent over the complex number field $C$, and $a_{1}, a_{2}, \ldots, a_{k}$ are nonzero constants. By (2.1) and (2.4), we have

$$
\sum_{i=1}^{k} a_{i} f_{i}+f_{n+1} \equiv 1
$$

By Lemma 5, (2.2) and (2.5) we can easily verify that $f_{1}, f_{2}, \ldots, f_{k}, f_{n+1}$ are linearly dependent over the complex number field $C$, hence we have

$$
c_{1} f_{1}+c_{2} f_{2}+\cdots+c_{k} f_{k}+c_{k+1} f_{n+1}=0,
$$

where $c_{1}, c_{2}, \ldots, c_{k}, c_{k+1}$ are constants not all equal to zero. Noting that 
$f_{1}, f_{2}, \ldots, f_{k}$ are linearly independent over the complex number field $C$, we can see that $c_{k+1} \neq 0$. From (2.6) we have

$$
f_{n+1}=-\frac{c_{1}}{c_{k+1}} f_{1}-\cdots-\frac{c_{k}}{c_{k+1}} f_{k}
$$

substituting (2.7) into (2.5) we get

$$
\sum_{i=1}^{k}\left(a_{i}-\frac{c_{i}}{c_{k+1}}\right) f_{i} \equiv 1 .
$$

From (2.8) we can see that $a_{i}-\frac{c_{i}}{c_{k+1}}(i=1,2, \ldots, k)$ are not all equal to zero. By Lemma 5, (2.2) and (2.8) we can have a contradiction. Thus, $\sum_{j=1}^{n} f_{j} \equiv 0$, and $f_{n+1} \equiv 1$, which proves Lemma 6 .

In 1999, H. X. Yi [11] proved the following result, which is an extension of Lemma 6: Let $f_{1}, f_{2}, \ldots, f_{n}$ be non-constant meromorphic functions, and let $f_{n+1}, f_{n+2}, \ldots, f_{n+m}$ be meromorphic functions such that

$$
f_{k} \not \equiv 0 \quad(k=n+1, n+2, \ldots, n+m)
$$

and

$$
\sum_{i=1}^{n+m} f_{i} \equiv A,
$$

where $A$ is a nonzero constant. If there exists a subset $I \subseteq R^{+}$satisfying mes $I=\infty$ such that

$$
\begin{gathered}
\sum_{i=1}^{n+m} N\left(r, \frac{1}{f_{i}}\right)+(n+m-1) \sum_{\substack{i=1 \\
i \neq j}}^{n+m} \bar{N}\left(r, f_{i}\right)<(\lambda+o(1)) T\left(r, f_{j}\right) \\
(r \rightarrow \infty, r \in I, j=1,2, \ldots, n),
\end{gathered}
$$

where $\lambda<1$. Then there exist $t_{i} \in\{0,1\}(i=1,2, \ldots, m)$ such that

$$
\sum_{i=1}^{m} t_{i} f_{n+i} \equiv A
$$

Lemma 7 (see [5, Theorem 1]). Let $f$ and $g$ be two distinct non-constant meromorphic functions sharing 0,1 and $\infty \mathrm{CM}$. If

$$
\limsup _{\substack{r \rightarrow \infty \\ r \notin E}} \frac{N_{0}(r)}{T(r, f)}>\frac{1}{2},
$$

then $f$ is a Möbius transformation of $g$. 
Lemma 8 (see [5, Theorem 2]). Let $f$ and $g$ be two non-constant meromorphic functions sharing 0,1 and $\infty \mathrm{CM}$. If

$$
0<\limsup _{\substack{r \rightarrow \infty \\ r \notin E}} \frac{N_{0}(r)}{T(r, f)} \leq \frac{1}{2},
$$

then $f$ is not any Möbius transformation of $g$, and $f$ and $g$ satisfy one of the following relations:

(i) $f \equiv \frac{e^{s \gamma}-1}{e^{(k+1) \gamma}-1}, g \equiv \frac{e^{-s \gamma}-1}{e^{-(k+1) \gamma}-1}$,

(ii) $f \equiv \frac{e^{(k+1) \gamma}-1}{e^{(k+1-s) \gamma}-1}, g \equiv \frac{e^{-(k+1) \gamma}-1}{e^{-(k+1-s) \gamma}-1}$,

(iii) $f \equiv \frac{e^{s \gamma}-1}{e^{-(k+1-s) \gamma}-1}, g \equiv \frac{e^{-s \gamma}-1}{e^{(k+1-s) \gamma}-1}$,

where $s$ and $k(\geq 2)$ are positive integers such that $1 \leq s \leq k$, and $s$ and $k+1$ are relatively prime, and $\gamma$ is a non-constant entire function.

LEMma 9 (see [4, Lemma 1]). Let $f$ and $g$ be two distinct nonconstant meromorphic functions sharing 0,1 and $\infty \mathrm{CM}$, then there exist two entire functions $\alpha$ and $\beta$ such that

$$
f \equiv \frac{e^{\alpha}-1}{e^{\beta}-1}, \quad g \equiv \frac{e^{-\alpha}-1}{e^{-\beta}-1},
$$

where $e^{\beta} \not \equiv 1, e^{\alpha} \not \equiv 1$ and $e^{\beta-\alpha} \not \equiv 1$, and

$$
T(r, g)+T\left(r, e^{\alpha}\right)+T\left(r, e^{\beta}\right)=O(T(r, f)) \quad(r \notin E) .
$$

LEMma 10 (see [12, Lemma 2.4]). Let $h$ be a non-constant meromorphic function and let $\alpha, \beta, \gamma$ be meromorphic functions such that $T(r, \alpha)+T(r, \beta)+$ $T(r, \gamma)=S(r, h)$, and $\alpha \neq \equiv 0$ or $\gamma \neq \equiv 0$. Furthermore, let

$$
H=\alpha h^{2}+\beta h+\gamma .
$$

If $\bar{N}(r, h)=S(r, h), \bar{N}(r, 1 / h)=S(r, h)$ and $N_{1)}(r, 1 / H)=S(r, h)$, then $\beta^{2}-4 \alpha \gamma \equiv 0$.

\section{Proof of Theorem 1}

By the assumptions of Theorem 1, we have

$$
\frac{f^{\prime}-a}{g^{\prime}-a}=e^{\delta},
$$

where $\delta$ is an entire function. Suppose that $e^{\delta} \equiv A$, where $A$ is a nonzero constant. From (3.1) we get

$$
f-A g=(1-A) a z+C,
$$


where $C$ is a constant. Since $f \not \equiv g$, from (3.2) we know that

$$
(1-A) a z+C \not \equiv 0 \text {. }
$$

By (3.2) and (3.3) we get

$$
\delta_{1)}(0, f)+\delta_{1)}(1, f)=2 .
$$

By Lemma 1, we have $f+g \equiv 1$ and $f^{\prime}+g^{\prime} \equiv 0$, which implies that $a$ is a Picard value of $f^{\prime}$ and $g^{\prime}$. This contradicts Hayman's inequality (see [1, Theorem 3.5]). Thus $e^{\delta}$ is not a constant, and hence

$$
\delta^{\prime} \not \equiv 0 .
$$

By logarithmic differentiation, from (3.1) we obtain

$$
\delta^{\prime}=\frac{f^{\prime \prime}}{f^{\prime}-a}-\frac{g^{\prime \prime}}{g^{\prime}-a} .
$$

By Lemma 2, (3.4) and (3.5), we get

$$
N_{1)}(r, f) \leq N\left(r, \frac{1}{\delta^{\prime}}\right) \leq T\left(r, \delta^{\prime}\right)+O(1)=S(r, f) .
$$

By Lemma 3, we have

$$
N_{(2}(r, f)=S(r, f) .
$$

By (3.6) and (3.7), we obtain

$$
N(r, f)=S(r, f) .
$$

We discuss the following two cases.

CASE 1. Suppose that $f$ is a Möbius transformation of $g$. By Lemma 4, we know that $f$ and $g$ satisfy one of the six relations in Lemma 4.

Assume that $f$ and $g$ satisfy the relation (i) in Lemma 4. Let $f=e^{\alpha}$, where $\alpha$ is a non-constant entire function. Then $g=e^{-\alpha}$. Substituting $f$ and $g$ into (3.1) we get

$$
\frac{\alpha^{\prime} e^{2 \alpha}-a e^{\alpha}}{-\alpha^{\prime}-a e^{\alpha}}=e^{\delta}
$$

By (3.9) we have

$$
T\left(r, e^{\delta}\right) \geq T\left(r, e^{\alpha}\right)+S(r, f)
$$

and

$$
\frac{\alpha^{\prime}}{a} e^{\alpha}+e^{\delta}+\frac{\alpha^{\prime}}{a} e^{\delta-\alpha} \equiv 1
$$

By Lemma 6, (3.10) and (3.11) we obtain 


$$
\frac{\alpha^{\prime}}{a} e^{\delta-\alpha} \equiv 1, \quad \frac{\alpha^{\prime}}{a} e^{\alpha}+e^{\delta} \equiv 0 .
$$

From (3.12) we get $\alpha(z)=a \omega z+C$, where $\omega$ satisfying $\omega^{2}=-1$, and $C$ are constants. Thus $f(z)=A e^{a \omega z}$ and $g(z)=(1 / A) e^{-a \omega z}$, where $A$ is a nonzero constant. From this we have the relation (i) in Theorem 1.

Assume that $f$ and $g$ satisfy the relation (ii) in Lemma 4. In the same manner as above, we can obtain $f(z)=1+A e^{a \omega z}$ and $g(z)=1+(1 / A) e^{-a \omega z}$, where $\omega$ satisfying $\omega^{2}=-1$, and $A(\neq 0)$ are constants. From this we have the relation (ii) in Theorem 1 .

Assume that $f$ and $g$ satisfy the relation (vi) in Lemma 4. In the same manner as above, we can obtain $f(z)=1 /(c-1)\left(A e^{a(c-1) \omega z}-1\right)$ and $g(z)=$ $c /(c-1)\left(1-(1 / A) e^{-a(c-1) \omega z}\right)$, where $\omega$ satisfying $\omega^{2}=1 / c$, and $A(\neq 0)$ are constants. From this we have the relation (iii) in Theorem 1 .

Assume that $f$ and $g$ satisfy the relation (iii) in Lemma 4 . Since $f$ and $g$ share 0,1 and $\infty \mathrm{CM}$, from the relation (iii) in Lemma 4, we know that 0 and 1 are Picard values of $f$. Thus $N(r, f)=T(r, f)+S(r, f)$, which contradicts (3.8).

Assume that $f$ and $g$ satisfy the relations (iv) and (v) in Lemma 4. In the same manner as above, we can obtain contradictions.

Case 2. Suppose that $f$ is not any Möbius transformation of $g$. By Lemma 7, we consider the following two subcases.

SubCASE 2.1. Assume that

$$
0<\limsup _{\substack{r \rightarrow \infty \\ r \notin E}} \frac{N_{0}(r)}{T(r, f)} \leq \frac{1}{2} .
$$

By Lemma 8, we know that $f$ and $g$ satisfy one of the three relations in Lemma 8.

Assume that $f$ and $g$ satisfy the relation (i) in Lemma 8. Then we have $N(r, f)=T(r, f)+S(r, f)$, which contradicts (3.8).

Assume that $f$ and $g$ satisfy the relation (ii) in Lemma 8. By (3.8) we know that $k=s$. Thus,

$$
f=e^{k \gamma}+e^{(k-1) \gamma}+\cdots+1, \quad g=e^{-k \gamma}+e^{-(k-1) \gamma}+\cdots+1 .
$$

By (3.13) we obtain

$$
T(r, f)=k T\left(r, e^{\gamma}\right)+S(r, f), \quad T(r, g)=k T\left(r, e^{\gamma}\right)+S(r, f) .
$$

Substituting (3.13) into (3.1) we get

$$
\frac{k \gamma^{\prime} e^{2 k \gamma}+(k-1) \gamma^{\prime} e^{(2 k-1) \gamma}+\cdots+\gamma^{\prime} e^{(k+1) \gamma}-a e^{k \gamma}}{-k \gamma^{\prime}-(k-1) \gamma^{\prime} e^{\gamma}-\cdots-\gamma^{\prime} e^{(k-1) \gamma}-a e^{k \gamma}}=e^{\delta} .
$$

By (3.14) and (3.15) we have 


$$
T\left(r, e^{\delta}\right) \geq k T\left(r, e^{\gamma}\right)+S(r, f)
$$

and

$$
\begin{aligned}
& \frac{k \gamma^{\prime}}{a} e^{k \gamma}+\frac{(k-1) \gamma^{\prime}}{a} e^{(k-1) \gamma}+\cdots+\frac{\gamma^{\prime}}{a} e^{\gamma}+e^{\delta} \\
& +\frac{\gamma^{\prime}}{a} e^{\delta-\gamma}+\frac{2 \gamma^{\prime}}{a} e^{\delta-2 \gamma}+\cdots+\frac{k \gamma^{\prime}}{a} e^{\delta-k \gamma} \equiv 1 .
\end{aligned}
$$

By Lemma 6, (3.14), (3.16) and (3.17) we obtain

$$
\frac{k \gamma^{\prime}}{a} e^{\delta-k \gamma} \equiv 1
$$

and hence

$$
e^{\delta} \equiv \frac{a}{k \gamma^{\prime}} e^{k \gamma}
$$

Substituting (3.18) into (3.17) we get

$$
\left(\frac{k \gamma^{\prime}}{a}+\frac{a}{k \gamma^{\prime}}\right) e^{k \gamma}+\left(\frac{(k-1) \gamma^{\prime}}{a}+\frac{1}{k}\right) e^{(k-1) \gamma}+\cdots+\left(\frac{\gamma^{\prime}}{a}+\frac{k-1}{k}\right) e^{\gamma} \equiv 0 .
$$

From (3.19) we obtain

$$
\frac{k \gamma^{\prime}}{a}+\frac{a}{k \gamma^{\prime}} \equiv 0, \quad \frac{(k-1) \gamma^{\prime}}{a}+\frac{1}{k} \equiv 0, \quad \frac{\gamma^{\prime}}{a}+\frac{k-1}{k} \equiv 0 .
$$

From (3.20) we have a contradiction.

Assume that $f$ and $g$ satisfy the relation (iii) in Lemma 8. By (3.8) we know that $s=k$. Thus,

$$
f=-e^{k \gamma}-e^{(k-1) \gamma}-\cdots-e^{\gamma}, \quad g=-e^{-k \gamma}-e^{-(k-1) \gamma}-\cdots-e^{-\gamma} .
$$

In the same manner as above, we can obtain a contradiction.

SubCASE 2.2. Assume that

$$
\limsup _{\substack{r \rightarrow \infty \\ r \notin E}} \frac{N_{0}(r)}{T(r, f)}=0 .
$$

Thus,

$$
N_{0}(r)=S(r, f) .
$$

Noting that $f$ and $g$ share 0,1 and $\infty \mathrm{CM}$, by Lemma 9 we have (2.9) and (2.10). If $e^{\beta} \equiv C$, where $C$ is a nonzero constant. From (2.9) we obtain

$$
\frac{(f-1) g}{f(g-1)} \equiv C
$$


From this we get that $f$ is a Möbius transformation of $g$, which is a contradiction. Thus, $e^{\beta}$ is not a constant. From (2.9) we obtain

$$
f-g=\frac{\left(e^{\alpha}-1\right)\left(1-e^{\beta-\alpha}\right)}{e^{\beta}-1} .
$$

We use $N_{0}^{*}(r)$ to denote the counting function of the common zeros of $e^{\alpha}-1$ and $e^{\beta}-1$. From (3.22), the following formula is obviously

$$
N_{0}(r)=N_{0}^{*}(r)+S(r, f) \text {. }
$$

From this and (3.21) we have

$$
N_{0}^{*}(r)=S(r, f) .
$$

By (2.9) and (3.23), we have

$$
N(r, f)=N\left(r, \frac{1}{e^{\beta}-1}\right)+S(r, f) .
$$

By (3.8) and (3.24) we get

$$
T\left(r, e^{\beta}\right)=S(r, f) .
$$

From (2.9) and (3.25) we have

$$
T(r, f)=T\left(r, e^{\alpha}\right)+S(r, f), \quad T(r, g)=T(r, f)+S(r, f) .
$$

Substituting (2.9) into (3.1) we get

$$
\frac{\left(\alpha^{\prime} e^{\beta}-\beta^{\prime} e^{\beta}-\alpha^{\prime}\right) e^{2 \alpha}+\left(\beta^{\prime} e^{\beta}-a e^{2 \beta}+2 a e^{\beta}-a\right) e^{\alpha}}{\left(\alpha^{\prime} e^{2 \beta}+\beta^{\prime} e^{\beta}-\alpha^{\prime} e^{\beta}\right)-\left(\beta^{\prime} e^{\beta}+a e^{2 \beta}-2 a e^{\beta}+a\right) e^{\alpha}}=e^{\delta} .
$$

It is obvious that

$$
\beta^{\prime} e^{\beta}-a e^{2 \beta}+2 a e^{\beta}-a \not \equiv 0, \quad \beta^{\prime} e^{\beta}+a e^{2 \beta}-2 a e^{\beta}+a \not \equiv 0 .
$$

If $\alpha^{\prime} e^{\beta}-\beta^{\prime} e^{\beta}-\alpha^{\prime} \equiv 0$, then

$$
\alpha^{\prime}=\frac{\beta^{\prime} e^{\beta}}{e^{\beta}-1} .
$$

By integration, from (3.29) we obtain

$$
e^{\alpha}=C\left(e^{\beta}-1\right),
$$

where $C$ is a nonzero constant, which is a contradiction. Thus

$$
\alpha^{\prime} e^{\beta}-\beta^{\prime} e^{\beta}-\alpha^{\prime} \not \equiv 0 \text {. }
$$

In the same manner as above, we have

$$
\alpha^{\prime} e^{2 \beta}+\beta^{\prime} e^{\beta}-\alpha^{\prime} e^{\beta} \not \equiv 0 .
$$

By (3.25), (3.26), (3.27), (3.28), (3.31) and (3.32) we have

$$
T\left(r, e^{\delta}\right) \geq T\left(r, e^{\alpha}\right)+S(r, f)
$$


and

$$
\begin{aligned}
& -\frac{\alpha^{\prime} e^{\beta}-\beta^{\prime} e^{\beta}-\alpha^{\prime}}{\beta^{\prime} e^{\beta}-a e^{2 \beta}+2 a e^{\beta}-a} e^{\alpha}-\frac{\beta^{\prime} e^{\beta}+a e^{2 \beta}-2 a e^{\beta}+a}{\beta^{\prime} e^{\beta}-a e^{2 \beta}+2 a e^{\beta}-a} e^{\delta} \\
& +\frac{\alpha^{\prime} e^{2 \beta}+\beta^{\prime} e^{\beta}-\alpha^{\prime} e^{\beta}}{\beta^{\prime} e^{\beta}-a e^{2 \beta}+2 a e^{\beta}-a} e^{\delta-\alpha} \equiv 1 .
\end{aligned}
$$

By Lemma 6, (3.25), (3.26), (3.33) and (3.34) we obtain

$$
\frac{\alpha^{\prime} e^{2 \beta}+\beta^{\prime} e^{\beta}-\alpha^{\prime} e^{\beta}}{\beta^{\prime} e^{\beta}-a e^{2 \beta}+2 a e^{\beta}-a} e^{\delta-\alpha} \equiv 1,
$$

and

$$
\frac{\alpha^{\prime} e^{\beta}-\beta^{\prime} e^{\beta}-\alpha^{\prime}}{\beta^{\prime} e^{\beta}-a e^{2 \beta}+2 a e^{\beta}-a} e^{\alpha}+\frac{\beta^{\prime} e^{\beta}+a e^{2 \beta}-2 a e^{\beta}+a}{\beta^{\prime} e^{\beta}-a e^{2 \beta}+2 a e^{\beta}-a} e^{\delta} \equiv 0 .
$$

From (3.35) and (3.36) we get

$$
\frac{\left(-\alpha^{\prime} e^{\beta}+\beta^{\prime} e^{\beta}+\alpha^{\prime}\right)\left(\alpha^{\prime} e^{2 \beta}+\beta^{\prime} e^{\beta}-\alpha^{\prime} e^{\beta}\right)}{\left(\beta^{\prime} e^{\beta}+a e^{2 \beta}-2 a e^{\beta}+a\right)\left(\beta^{\prime} e^{\beta}-a e^{2 \beta}+2 a e^{\beta}-a\right)} \equiv 1 .
$$

From (3.37) we obtain

$$
e^{\beta}\left(\alpha^{\prime}-\frac{\beta^{\prime}}{2}\right)^{2} \equiv a^{2} e^{2 \beta}+\left(\frac{\left(\beta^{\prime}\right)^{2}}{4}-2 a^{2}\right) e^{\beta}+a^{2}
$$

Set

$$
H=a^{2} e^{2 \beta}+\left(\frac{\left(\beta^{\prime}\right)^{2}}{4}-2 a^{2}\right) e^{\beta}+a^{2}
$$

then

$$
H=e^{\beta}\left(\alpha^{\prime}-\frac{\beta^{\prime}}{2}\right)^{2}
$$

Applying Lemma 10 to $H$, from (3.39) and (3.40) we have

$$
\left(\frac{\left(\beta^{\prime}\right)^{2}}{4}-2 a^{2}\right)^{2}-4 a^{4} \equiv 0
$$

From (3.41) we get

$$
\beta^{\prime}=4 a \omega
$$

and hence

$$
e^{\beta}=A e^{4 a \omega z}
$$


where $\omega$ satisfying $\omega^{2}=1$, and $A$ are nonzero constants. Substituting (3.42) and (3.43) into (3.38), we have

$$
\alpha^{\prime}=2 a \omega+a \omega_{1}\left(B_{1} e^{2 a \omega z}+\frac{1}{B_{1}} e^{-2 a \omega z}\right),
$$

where $B_{1}$ and $\omega_{1}$ are constants satisfying $B_{1}^{2}=A$ and $\omega_{1}^{2}=1$. Thus,

$$
\alpha=2 a \omega z+\frac{\omega_{1}}{2 \omega}\left(B_{1} e^{2 a \omega z}-\frac{1}{B_{1}} e^{-2 a \omega z}\right)+C,
$$

where $C$ is a constant. Set $B=\frac{\omega_{1} B_{1}}{\omega}$, then $B^{2}=A$ and

$$
\alpha=2 a \omega z+\frac{1}{2}\left(B e^{2 a \omega z}-\frac{1}{B} e^{-2 a \omega z}\right)+C .
$$

From (3.45) we have

$$
\alpha^{\prime}=2 a \omega+B a \omega \cdot e^{2 a \omega z}+\frac{a \omega}{B} \cdot e^{-2 a \omega z} .
$$

Noting that $B^{2}=A$, from (3.42), (3.43) and (3.46) we get

$$
\alpha^{\prime} e^{2 \beta}+\beta^{\prime} e^{\beta}-\alpha^{\prime} e^{\beta}=B^{5} a \omega \cdot e^{10 a \omega z}+2 B^{4} a \omega \cdot e^{8 a \omega z}+2 B^{2} a \omega \cdot e^{4 a \omega z}-B a \omega \cdot e^{2 a \omega z}
$$

and

$$
\beta^{\prime} e^{\beta}-a e^{2 \beta}+2 a e^{\beta}-a=(4 a \omega+2 a) B^{2} \cdot e^{4 a \omega z}-a B^{4} \cdot e^{8 a \omega z}-a .
$$

Substituting (3.47) and (3.48) into (3.35), we deduce

$$
\frac{e^{8 a \omega z}-(4 \omega+2) / B^{2} \cdot e^{4 a \omega z}+1 / B^{4}}{e^{8 a \omega z}+2 / B \cdot e^{6 a \omega z}+2 / B^{3} \cdot e^{2 a \omega z}-1 / B^{4}} \equiv-B \omega e^{\delta-\alpha+2 a \omega z} .
$$

Let

$$
P_{1}(\chi)=\chi^{8}-\frac{4 \omega+2}{B^{2}} \cdot \chi^{4}+\frac{1}{B^{4}}, \quad P_{2}(\chi)=\chi^{8}+\frac{2}{B} \cdot \chi^{6}+\frac{2}{B^{3}} \cdot \chi^{2}-\frac{1}{B^{4}} .
$$

From (3.50) we can easily see that every root of $P_{j}(\chi)=0(j=1,2)$ is not equal to zero, and that there is at least one root of $P_{1}(\chi)=0$ that is not any root of $P_{2}(\chi)=0$. Thus, from (3.49) we can have a contradiction.

Theorem 1 is thus completely proved.

Acknowledgement. The authors want to express their thanks to the anonymous referee for his valuable suggestions and comments. 


\section{REFERENCES}

[ 1] W. K. Hayman, Meromorphic Functions, Oxford Math. Monogr., Clarendon Press, Oxford, 1964.

[2] H. X. Yi AND C. C. YANG, Uniqueness Theory of Meromorphic Functions, Science Press, Beijing, 1995.

[3] K. TohgE, Meromorphic functions covering certain finite sets at the same points, Kodai Math. J., 11 (1988), 249-279.

[ 4 ] H. X. YI, Unicity theorems for meromorphic functions that share three values, Kodai Math. J., 18 (1995), 300-314.

[5] Q. C. ZhANG, Meromorphic functions sharing three values, Indian J. Pure Appl. Math., 30 (1999), 667-682.

[6] Amer H. H. Al-Khaladi, Unicity theorems for meromorphic functions, Kodai Math. J., 23 (2000), 27-34.

[7] Q. C. Zhang, Meromorphic functions sharing values or sets, Doctoral Thesis, Shandong University, 1999.

[8] R. Nevanlinna, Le Théorème de Picard-Borel et la Théorie des Fonctions Méromorphes, Gauthier-Villars, Paris, 1929.

[9] P. LI AND C.-C. YANG, Some further results on the unique range sets of meromorphic functions, Kodai Math. J., 18 (1995), 437-450.

[10] H. X. YI, Some theorems on systems of meromorphic functions, J. Shandong Univ. Nat. Sci. Ed., 32 (1997), 121-127.

[11] H. X. YI, Some theorems on systems of meromorphic functions III, J. Shandong Univ. Nat. Sci. Ed., 34 (1999), 1-9.

[12] P. Li AND C.-C. YANG, When an entire function and its linear differential polynomial share two values, Illinois J. Math., 44 (2000), 349-362.

Department of Mathematics

SHANDONG UNIVERSITY

JiNAN, SHANDONG 250100

PeOPLE's RePublic of China

e-mail: li-xiaomin@163.com

Department of Mathematics

SHANDONG UNIVERSITY

JinAN, Shandong 250100

People's Republic of China

e-mail: hxyi@sdu.edu.cn 